\title{
Spontaneous adrenal hemorrhage during pregnancy: \\ a case with horseshoe kidney
}

\author{
Mohadeseh Amini, Meysam Moghbeli \\ North Khorasan University of Medical Sciences, Bojnurd, Iran
}

\begin{abstract}
Spontaneous adrenal hemorrhage is an acute hemorrhage during pregnancy, which can be tragic for the mother and the baby. We report a unique spontaneous hemorrhage during pregnancy in a case with horseshoe kidney with separated adrenal, presented for the first time in the world. Computed tomography scan showed a horseshoe kidney fused with left normal kidney. Interestingly the adrenal gland was remained in right flank and separated from the horseshoe kidney, which prepares a probable physical stress for the hemorrhage. Diagnosis and surgery were done successfully and the case was fully recovered after several days.
\end{abstract}

Correspondence: Meysam Moghbeli, North Khorasan University of Medical Sciences, P.O. Box: 74877-94149 Bojnurd, Iran.

Tel.: +98.915.151.4405

E-mail:m.moghbeli@nkums.ac.ir ;

meysam_moghbeli@yahoo.com

Key words: Adrenal; horseshoe kidney; spontaneous hemorrhage; pregnancy.

Conflict of interest: the authors declare no conflict of interest.

Informed consent: informed consent form which was approved by the Ethics Committee of North Khorasan University of Medical Sciences was obtained from the patient.

Ethical standards: this study has been approved by the appropriate Ethics Committee and has therefore been performed in accordance with the ethical standards laid down in the 1964 Declaration of Helsinki.

Received for publication: 5 April 2017.

Revision received: 2 July 2017.

Accepted for publication: 5 July 2017.

This work is licensed under a Creative Commons Attribution NonCommercial 4.0 License (CC BY-NC 4.0).

(C) Copyright M. Amini and M. Moghbeli, 2017

Licensee PAGEPress, Italy

Italian Journal of Medicine 2017; 11:415-416

doi:10.4081/itjm.2017.872

\section{Imaging in Internal Medicine}

Adrenal hemorrhage has a mortality rate of $15 \%$, which is due to the capsule rupture. ${ }^{1,2}$ Retroperitoneal hematoma following the adrenal hemorrhage during pregnancy is a rare complication. We presented an interesting pregnant case with unilateral adrenal hemorrhage that had a horseshoe kidney in left flank. A pregnant 34-year-old female with gravid two/live two who claimed about sudden severe abdominal pain was referred to the Emergengy Department one day after the natural childbirth. Abdominal examination showed right abdominal tenderness, nausea, and vomiting. Blood pressure pulse rate, hemoglobin, and body temperature were $100 / 70,80,10.6$, and $36.5^{\circ} \mathrm{C}$, respectively. Intravenous therapy was performed and abdominal/pelvic sonography showed a heterogenic area in right paracolic gutter with free fluid in Morison's pouch, pelvic, and inter loop. Right kidney was not observed in the normal location. Uterus was heterogenic with a size of higher than the normal status. Endometrial thickness was reported as seven millimeters. Computed tomography scan showed a peritoneal hematoma in right flank with a horseshoe kidney in an abnormal location (Figure 1). The patient was pale

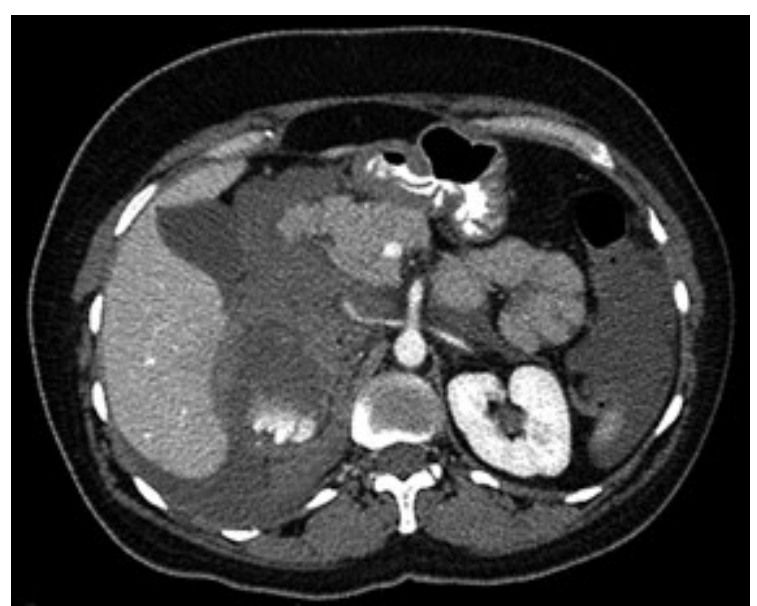

Figure 1. Computed tomography scan of abdomen and pelvis. 
with shock and referred to the Intensive Care Unit for the rehabilitation. Laparotomy was performed to clump the sub diaphragm aorta. Patient was undergone the blood transfusion, rehabilitation, and central venous catheter insertion. Right uterine artery was ligated following the massive bilateral retroperitoneal hematoma. Adrenal artery was also ligated following the sub-hepatic bleeding. The patient was discharged after 10 days with a normal status. This was a unique case for the first time in a pregnant female, indicating the role of kidney in preserving the normal status of adrenal gland. Indeed, separated adrenal gland will be faced with more physical tensions during the different physical activities and specifically during pregnancy.

\section{References}

1. McGowan-Smyth S. Bilateral adrenal haemorrhage leading to adrenal crisis. BMJ Case Rep 2014;2014: pii: bcr2014204225.

2. Souiki T, Tekni Z, Laachach H, et al. Catastrophic hemorrhage of adrenal pheochromocytoma following thrombolysis for acute myocardial infarction: case report and literature review. World J Emerg Surgery 2014;9:50. 